\title{
Gaps and Barriers to Successful Integration and Adoption of Practical Materials Informatics Tools and Workflows
}

\author{
DAVID L. MCDOWELL ${ }^{1,2,3}$ \\ 1.-Woodruff School of Mechanical Engineering, Atlanta, USA. 2.-School of Materials Science \\ and Engineering, Georgia Institute of Technology, Atlanta, GA 30332, USA. \\ 3.-e-mail: david.mcdowell@me.gatech.edu
}

Preparation of the future materials workforce consistent with major imperatives rooted in integrated computational materials engineering (ICME) and the materials genome initiative (MGI) is most effectively pursued within the vision of a materials innovation ecosystem that spans across conventional engineering, science, and computing disciplines. The ICME foundation integrates principles of materials science with computational methods, including increasing reliance on modern data science methods that are savvy to digital information that recognizes hierarchical material structure and the need for correlative relations for process-structure and structure-property relations. We consider gaps in academic research and education programs related to systems engineering, uncertainty quantification of both experiments and computation, and data science methods. Barriers to the introduction of materials data science are discussed, as well as opportunities for innovation in educating the future MGI and ICME workforce.

\section{INTRODUCTION}

Materials science and engineering (MSE) was established as an academic discipline in the latter half of the twentieth century. Particularly in the last 10 years, new initiatives have taken hold that have been embraced by industry and the research community and require the leveraging of advances in computing and modern data science: integrated computational materials engineering (ICME) and the Materials Genome Initiative (MGI). These initiatives serve as harbingers of future workforce needs in materials discovery, development, and product deployment. As in all branches of economics, business, science, and engineering, modern materials science is compelled to effectively integrate modern data science with the digital information which is so prevalent in this field to enhance productivity and increase the rate of progress in R\&D. Broadly speaking, the materials

(Received July 14, 2020; accepted October 5, 2020; published online October 26, 2020) industry, government, and academic cohorts are still in the early stages of responding to this imperative.

The ICME initiative emerged from a 2008 National Academy of Engineering National Materials Advisory Board report. ${ }^{1}$ ICME addresses the historical difficulty in bridging the gap that exists between new and improved material concepts developed in basic research and their deployment into products, the so-called valley of death. ICME represents an approach to the design and development of materials that considers product requirements and chiefly focuses on integration of modeling and simulation at various length and time scales with materials synthesis, processing, characterization, and property measurements to provide enhanced, more timely decision support. The 2011 Materials Genome Initiative (MGI) ${ }^{2}$ endures as a federal initiative that very much complements ICME. The MGI strategic plan, issued in December $2014^{3}$ by the subcommittee on the MGI tasked by the National Science and Technology Council, encompasses both materials discovery and development aspects, and requires joint investment by the science and engineering communities. 
ICME and MGI reinforce the need for digital materials science and engineering, and recognize the pivotal role of data science in this regard, as discussed in Ref. 4. The present article considers gaps and barriers with regard to the successful integration and adoption of practical materials informatics tools and associated computational/digital workflows. The author has devoted nearly a decade to visioning and supporting the development of research initiatives that incorporate materials data science within the setting of a major research university, Georgia Tech, so perhaps this perspective can be of value to others. We start by discussing community identification of the skills needed for the future workforce, opportunities, and imperatives at the intersection of materials and manufacturing.

\section{WHAT SKILLS WILL THE FUTURE MATERIALS WORKFORCE NEED?}

In addition to acknowledging the role of computation and digital data as foundational to both ICME and the MGI, the latter has particularly emphasized data science as an essential component of the acceleration of R\&D. The addition of data science has been greatly reinforced with emerging emphasis over the past 5 years on artificial intelligence, machine learning, and methods for uncertainty quantification. We are clearly at a crossroads of technologies that are shaping every aspect of business, commerce, and communication, and will similarly "punctuate the equilibrium" status of materials and manufacturing. Academia must acknowledge this shift. To this end, education and training of materials researchers, practicing engineers, and technologists requires substantial thought and innovation. Over the next decade, it will be necessary to educate and train a materials workforce that will usher in this transformation for the next 20-30 years, aligned with ICME and the MGI. For example, the NASA Vision 2040: A Roadmap for Integrated, Multiscale Modeling and Simulation of Materials and Systems ${ }^{5}$ relies heavily on digital data, data science, computation, and digital workflows to engage academia, industry, and government to successfully pursue its goals.

Outcomes of the workshop Building an Integrated MGI Accelerator Network, ${ }^{6}$ held 5-6 June 2014 at Georgia Tech, provide a glimpse into the skillset needed by these future materials researchers and technologists. This workshop, co-organized by the Georgia Institute of Technology, the University of Wisconsin-Madison, and the University of Michigan, assembled 150 thought leaders and academics, and industry and government stakeholders from across the nation. Breakout sessions focused on critical MGI gaps and issues regarding materials for key application domains, including materials for organic and inorganic electronics, structural materials, materials for energy storage and conversion, biomaterials, and materials and interfaces for catalysis and separation. These sessions identified a set of common gaps across broad materials classes that must be addressed to effectively pursue integration of digital data, data science, computation, and experiments to accelerate the discovery and development of new and improved materials. We may view this list as comprising knowledge/skill gaps that must be addressed by the future workforce:

- Consideration of long-term stability under service conditions, environmental stability, degradation, and estimates of performance lifetime at early stages of discovery and development.

- Predictive simulation of metastable states and nonequilibrium trajectories of evolution under service conditions for applications, enabling parametric exploration of candidate material systems for product applications.

- Measurement science and modeling and simulation of synthesis and processing.

- Principles of kinetic and thermodynamic control of process route/structure relations. Exert reliable control of structure over various length scales (nano to macro) during processing, including up to large scales.

High-priority recommendations were identified at this workshop, the first of which was to focus on education and training of the future MGI workforce. Clearly this is an imperative for all of industry, government, and academia to address. Additional priority recommendations included, among others, linking physical and cyber infrastructure that cuts across materials classes and application domains, and establishing a distributed materials information infrastructure.

\section{HOW ARE MAJOR UNIVERSITY MATERIALS PROGRAMS ALIGNED WITH FUTURE WORKFORCE NEEDS?}

It is interesting and relevant to consider how leading materials science and engineering academic units convey their research and education interests in relation to these future workforce gaps and challenges. To this end, a casual review of websites of the top ten materials engineering graduate programs as ranked in 2020 by US News and World Report $^{7}$ (MIT, NWU, UC-Berkeley, Stanford, UCSB, UIUC, Caltech, University of Michigan, Georgia Tech, and Cornell) reveals a rather broad palette of approaches to representing the discipline to the external world. Most of these units expressed capabilities and aspirations largely in terms of classes of materials in which their faculty work (e.g., metals, ceramics, polymers, composites, dielectrics, etc.). A few of these units represented capabilities and aspirations more broadly in terms of opportunity areas for societal and economic impact. Very few discussed broader interests at the intersection of data science, computation, and 
experiments-the elements of the MGI. It seems that academic units may be comfortable thinking of their programs in a more traditional sense, rooted in frameworks established since the 1960s, augmented by nanotechnology and materials for biology since the late $1990 \mathrm{~s}$. As of yet, there is no clear trajectory for a common representation of material science that reflects ICME and MGI goals. Certainly, goals of selected research centers within US universities and laboratories do resonate closely with the MGI and ICME (e.g., the Institute for Materials at Georgia Tech (IMat), ${ }^{8}$ the Wisconsin Materials Institute, ${ }^{9}$ and the Materials Project at UC-Berkeley ${ }^{10}$ ), and the listing of interagency MGI activities on the NIST MGI site. ${ }^{11}$ However, these sites do not in themselves represent the academic future workforce perspective.

\begin{tabular}{|l|}
\hline \multicolumn{1}{|c|}{ MGI and ICME Messaging } \\
\hline Perhaps leading MSE academic units should \\
pursue strategies to organize and convey \\
research interests, capabilities and educational \\
aspirations in ways that address the \\
knowledge gaps and essential skillsets for \\
today's future workforce, which differs \\
substantially from those of prior generations. \\
\hline
\end{tabular}

\section{LEVERAGING THE CONVERGENCE OF MATERIALS AND MANUFACTURING}

A major trend has emerged and accelerated in the early twenty first century, namely the intersection of digital manufacturing with materials structure characterization, associated digital datasets, and computational materials science. Digital manufacturing involves the exploitation of an integrated, cyber-enabled system comprised of three-dimensional (3D) visualization, simulations, analytics, and various collaboration tools to simultaneously create product and manufacturing process definitions. ${ }^{12}$ The emergence of digital manufacturing coincides with the rapid expansion of digital characterization and workflows to support development of materials process-structure-property (PSP) relations. MGI and ICME are driving the linkage between the materials $R \& D$ and the materials supply chain more towards a focus on specifications of digital material structure, which relates to properties. ${ }^{4,13}$

Two major current trends can enhance consumer products, namely (i) digital manufacturing and reliable additive manufacturing and (ii) the concept of the digital twin. In each case, manufacturing of qualified products cannot be pursued without full, concurrent engagement of the materials $R \& D$ community. First consider additive manufacturing (AM). ${ }^{14,15}$ Since in this case material structure and properties are inextricably linked to the primary manufacturing process, materials must be tailored for PSP relations across the range of conditions to be encountered in the manufacturing processes; anticipation of qualification and certification requires materials engineering to be conducted concurrently with manufacturing. Given the interplay of part geometry, heterogeneity of built-up parts, distortion, and residual stresses, etc. with the material process route and material structure/microstructure, there are at least two ramifications: (a) the material process path and resulting microstructure should be captured as a part of the digital manufacturing data, which historically includes geometry, tolerancing, and metadata to guide machine control, and (b) data science is essential for tracking this information in seeking correlations related to downstream certification and qualification for required performance. Without coupling to predictive or correlative materials science, deployment of $\mathrm{AM}$ is tantamount to Edisonian trial and error; only commodity parts with little consequence of failure might even be considered in this case. Since predictive theory and computation are extremely limited in their capabilities to consider the evolution of material structure along such nonequilibrium process paths that produce gradients in chemistry and structure, there is no clear path forward in linking materials with additive manufacturing without employing materials data science and informatics. Even large-scale brute-force computation is not likely to deliver such a linkage (owing to uncertainty).

Secondly, consider the concept of the digital twin, ${ }^{16},{ }^{17}$ which serves as a virtual digital representation of a given physical system, such as a 3D model of an object or a collection of parts in subassemblies or assemblies. The digital twin purports to mimic not only geometry and tolerancing, but also in-service behavior and material degradation. It can potentially be used to simulate companion physical objects/systems in the digital computer, including sensor feedback from the actual system as a means of monitoring and controlling the response of the virtual representation. Invariably, the digital twin should faithfully represent all the salient physics associated with the system response, complex interactions, and even degradation or failure. This is also an application that places high demands on digital material representations and predictive computational simulation and/or correlations. As is the case with AM, it is difficult to see a path forward with high-performance computing based only on deterministic material models, absent some statistical learning protocols from data science to address uncertainty and incorporate information from physical measurements. 
Accordingly, these two emergent themes (and perhaps others) serve as excellent platforms on which to build case studies and examples for coupling materials design and development with manufacture. They also provide a compelling context in which to convey important educational concepts and themes in modern materials science and engineering, including thermodynamics, kinetics, process-structure-property relations, materials structure characterization, and uncertainty quantification and data science.

The author has emphasized the importance of both of these themes in chapter 6 of the Organization for Economic Co-operation and Development (OECD) report ${ }^{18}$ released at the G7 summit entitled The Next Production Revolution: An Overview of Key Issues and Policy Proposals. Specifically, chapter 6 of part 1 on key technologies identified New and Improved Materials and the Next Production Revolution as one of the foundational key technologies, along with digitalizing production, biomanufacturing, untapping nanotechnology's potential, 3D printing, and sustainability. As discussed in chapter 6, several transformational trends are accelerating the coupling of materials and manufacturing ${ }^{18}$ :

- The democratization of quantum mechanics and the ability to play "what-if" games to explore the potential performance of new and improved materials via predictive modeling and simulation. Computational quantum mechanics and atomistic modeling was initially the province of physics and chemistry, but now pervades engineering education and practice as a toolset to support materials design and development, even in disciplines such as mechanical, aerospace, and civil engineering, as well as the manufacturing sciences.

- Recognition of the hierarchy of material structure plays a pivotal role in digital representations of materials that support accelerated materials development, ranging from atoms (sub-nanometer) to molecules to interfaces between multiple phases.

- Along with the digital representation of hierarchical material structures, advances in highresolution materials characterization and in situ measurements facilitate the creation of digital workflows in support of digital manufacturing and design/optimization for desired property/ response targets.

- At the same time, manufacturing is embracing information regarding materials science, chemistry, and physics, enabling consideration of complex coupled effects of manufacturing processes with environment and service conditions.

- Digital workflows facilitate a traceable pedigree including the history of how materials are synthesized or processed, how their structure and properties are measured, and how they are used in specific manufactured products. Combined with materials information infrastructure (information theory, databases and data registries, digital interfaces, and distributed ecollaboration), this offers a powerful and economical approach to tracking the chain from materials supply to design and production.

- Formal theory and methods to support decisionmaking in materials development that considers the value of information in supporting design decisions and methods for uncertainty management, integrated with digital workflows.

\section{Digital Workflows are a Natural Bridge}

Placing emphasis on digital representations of material structure links to kinetics and thermodynamics through PSP relations, enabling new kinds of digital

representations of materials as the transactional basis for the supply chain to communicate available materials and processes, and new ways of archiving material pedigree and workflows.

Another transformational trend lies at the intersection of data science and materials. Both "big data" and "small data" are important in materials R\&D across the spectrum of discovery, development, and deployment. Some fields, such as weather forecasting, facial recognition, epidemiology, and medical diagnostics, rely on enormous volumes of data to inform high-quality correlations that enable reliable decision support. On the other hand, data in materials science are often expensive to acquire and extremely limited in both volume and velocity. Modern data science tools exist that deal with the value of information in scarce data environments and are able to provide meaningful guidance in identifying high-value datasets to acquire to improve decision support, including quantification of uncertainty in guiding decision-making based on limited data. These aspects of data science are critical for the materials workforce to learn and apply. They offer the potential to transform industry practice and should be addressed as a fundamental academic subject. We can foresee that, in the future, competitive research proposals will require this kind of approach to justify requested resources and proposed statements of work, which will of course draw the interest of academic researchers.

\section{OBSERVATIONS AND PRESSING NEEDS IN UNIVERSITIES}

Certain aspects of academic culture in materials education limit the rate at which advances can be made in addressing the educational needs of the data-savvy next-generation materials workforce. We next discuss barriers to the incorporation of 
materials informatics and digital materials science into the curriculum, based largely on personal observation and anecdotal information. In some cases, survey data are available.

University materials programs have largely shunned "complexity," favoring instead the presentation of information largely based on foundational principles and unit processes. Accordingly, the typical materials curriculum has little linkage to practical systems-level engineering, computational estimates, many-body effects, multiscale modeling and experiments, mesoscopic structures and responses, formal methods for uncertainty quantification, and scale-up manufacturing. This is rationalized by the perception of the immutable character of the "sheer volume" of foundational principles and concepts that any qualified materials scientist or engineer must ingest and master. We may think of this as an "empty vessel syndrome," the notion that only so much information can be poured in. This is a challenge common to many fields. While it is true that sequencing of information is critical to building understanding, human beings are not "fixed-volume containers" for knowledge; timely presentation of connections to other fields with brief but frequent illustrative examples or modules serve to feed the inquisitive nature of students, broaden perspectives, and expand knowledge. Availability of "easy to implement" modules interspersed at the right time in basic courses would go a long way to address variations in the experience and style of instructors.

Second, there is a lack of consistent reinforcement/use of computational methods, verification/validation, and uncertainty quantification (VVUQ), design-of-experiments methods, and principles of data science in coursework and design projects. A recent TMS study group report on Creating the Next-Generation Materials Genome Initiative Workforce ${ }^{19}$ found that computational materials science is offered in 40 of 50 sampled institutions' undergraduate or graduate materials science departments, but data science is present in only 9 of 50 institutions' undergraduate or graduate materials science departments. Based on personal experience at the undergraduate level, it is challenging to reinforce computational methods in MSE taught in sophomore or junior years, since a substantial number of faculty are not experienced in computation and numerical simulation.

Third, the materials research and education community has remained largely isolated from multidisciplinary design optimization and engineering systems communities rooted in other branches of engineering (e.g., mechanical and aerospace engineering, applied mechanics) where VVUQ and decision support are emphasized.

Finally, there is a lack of emphasis on underlying translational technologies and tools that cut across materials classes. Data science offers a logical means to "sew together" disparate materials and applications in expressing process-structure-property relations that are difficult to reconcile otherwise, which has led to compartmentalization of curricula. Using this strategy, UQ can be applied to PSP relations, and both relevant experimental methods and computational tools can be introduced and reinforced. It is conceivable that most of the materials curriculum could be integrated in this way using digital workflows and data science methods to unify tools and methods of learning and discovery.

Some of these barriers may naturally reduce with time as new faculty or instructors are hired, but that approach has a long timeframe and active dialog is preferred to generate understanding and buy-in. It is likely, as well as necessary, to carefully examine and design the range of concepts and technologies to which students should be exposed with expectations of "literacy." This may well require modular training, multimodal educational delivery (partially synchronous, partially asynchronous), and updating of laboratory and design courses to interplay with other academic units and leverage the preparation of students to address key gaps in materials discovery and development.

\section{THE PAUCITY OF VVUQ IN MATERIALS EDUCATION}

One particular aspect of engineering education in general, and materials education in particular, is woefully underemphasized. Uncertainty quantification (UQ) is a foundational, underemphasized aspect of modern materials science. Moreover, formal concepts of verification and validation are largely absent from the curriculum. UQ is primarily addressed by other engineering disciplines that move from basic unit process models to complex systems in design, for example, mechanical, aerospace, chemical and biomolecular, and civil engineering. On the other hand, a rich history of materials science textbooks effectively focus on model form uncertainty by pointing to various alternative or competing mechanisms or explanations for observed phenomena, on which novel curricula can capitalize. Furthermore, the material PSP relations offer perhaps one of the most obvious "templates" on which to systematically identify reducible and irreducible/random forms of uncertainty for instructional purposes.

Having interacted with engineering design and multidisciplinary design optimization groups over the years $\left(\mathrm{cf}^{20}{ }^{2}\right.$ ), it has become apparent that one distinction of materials design from other domains of design is the lack of sufficient quantitative models to enable formal design optimization for general PSP relations. Systems design approaches based on optimization require extensive models. While this may sometimes be configured in large-scale computational efforts at national laboratories or largescale funded R\&D programs with focused objectives, 
this kind of completeness of model coverage is normally lacking in materials PSP relations-there are often huge gaps, especially in process-structure relations. Some (many) of the "models" are based on empirical correlations or hierarchical multiscale models in space and/or time. While approximate surrogate models can be constructed to bridge these gaps and provide coverage to facilitate optimization, this requires careful consideration of the uncertainty of the approximation. Data science offers approaches to build such surrogates that minimize uncertainty for limited datasets. This points to a fundamental complication of applying data science methods to materials compared with fields that are dominated by "big data" (e.g., large volumes), which we categorize as the "big data" versus "small data" dichotomy. This was discussed earlier in this paper. The situation of "small data" in materials R\&D is a common scenario. This might lead one to erroneously conclude that data science has little bearing on materials $R \& D$, since the need for large datasets is commonly asserted in popular data science correlations. In materials $\mathrm{R} \& \mathrm{D}$, there is a pressing need for algorithms and methods that provide guidance for investing resources to acquire the next experimental dataset or conduct the next simulation to maximize the value of information gained relative to the cost. ${ }^{21}$ Similar kinds of optimization problems are commonly addressed in statistical learning methods that employ methods such as Gaussian regression with Bayesian updates. ${ }^{22}$

Research on complex ICME issues such as UQ for materials modeling across scales is now being actively pursued ( $\mathrm{cf}^{23}$ ), including contributions in data science and artificial intelligence/machine learning. Uncertainty associated with computational modeling across a wide range of length and time scales is certainly among the greatest challenges to reliable decision-making in materials design and development. Chapter 1 of Ref. 23 offers an overview of UQ methods in computational materials science, including key references. A study administered by the TMS is underway to consider how to accelerate the implementation of verification and validation in computational models that support decision-making in ICME. ${ }^{24}$

\section{THE IMPORTANCE OF DIGITAL WORKFLOWS}

Modern educational coursework routinely employs online digital "portals" or repositories for course information, including lectures, reference materials, learning objectives, and supplementary links. Digital course outlines and information repositories/registries are commonplace, even for courses otherwise offered face-to-face in the classroom. In addition, many courses are routinely offered in distance learning, on-line format, with asynchronous and/or synchronous lectures. This offers an ideal entry point for digital workflows-repetitive sequences of complex operations or transformations performed on datasets to accomplish desired goals or outcomes to guide decision-making and learning. One common universal theme for future workforce education and training is the need to understand, develop, and employ proficiency in constructing and manipulating digital workflows. Academic institutions can focus on open-source tools for building workflows and e-collaboration, ${ }^{25,26}$ as is done in modern business education and management. Such workflows ensure interoperability of research and education efforts to promote teaming. Second, they facilitate continuity of research protocols and products such as datasets and software from one generation of user to the next within an organization. Third, they enhance the transparency and reproducibility of research methodologies and results, including details of any approximations or assumptions made that are often not reported in various documents, including conference publications and journal articles. Finally, modular workflows can be repurposed for parallel tasks and efforts, enhancing efficiencies of reuse and scale-up. They can be used to present instructional materials and standardize the presentation of basic concepts, and certainly to reinforce joint use of computation, experiments, and data science, since correlations and statistical tools can be readily applied within digital workflows.

\section{Leveraging Today's Digital Classroom}

We will need to modify forms/modes of

presentation of instructional materials in the classroom and beyond, textbooks, and modules to digital modular format. Most universities are already using digital portals for courses, and this has been significantly accelerated and advanced with on-line offerings during the COVID-19 pandemic.

Students can implement and exercise these workflow scripting tools at early stages of their program as valuable assets to support the organization and integration of increasingly complex information and tasks into their coursework through capstone design. In research, such workflows will be at least as important as the data files themselves in documenting detailed procedures, including associated metadata. Moreover, they form a logical, effective, and efficient basis for establishing meaningful collaboration between research groups engaged in computation, or between computational groups and experimentalists. Entire digital pipelines for workflow sequences can be mapped and transferred, allowing nonexperts or novices to access and learn state-of-the-art methods rather than reinventing the wheel or relying on "home-built" codes and schema. 


\section{SOME KEY POINTS REGARDING MATERIALS EDUCATION AND TRAINING}

A number of engineering disciplines have leveraged the "democratization of quantum mechanics" to make inroads on the fusion of materials science and concurrent design of materials and products. They are becoming increasingly fluent in coupling materials and manufacturing in areas such as additive manufacturing, materials design, etc. Mechanical engineering, chemical and biomolecular engineering, industrial or manufacturing systems, and biomedical engineering are addressing systems integration in areas such as bioimplants and cell manufacturing. Within this context, the discussion regarding the role graduates of materials engineering and technology programs within the future workforce is a topic of high importance. Should key twenty first century exemplar technologies of materials discovery and development reside within or
- Students should be introduced to data science as a means of organizing and fusing information obtained from experiments, simulations, expert knowledge, literature, and other sources to support decision-making in materials R\&D. Materials data science is not at all synonymous with computational materials science, they are distinct and complementary, in a manner similar to materials data science and experiments.

- Undergraduates should receive more instructional emphasis on UQ, not just "statistics," with reinforcement in laboratory and design courses.

- Materials academic units may consider a rigorous graduate course in UQ for materials, as well as course modules in undergraduate required courses that introduce basic concepts and apply them in various topical domains.

The materials-manufacturing nexus is a very good exemplar for capstone design that reinforces all of

A Common (and Unfortunate) Misperception

Materials data science and informatics (and more broadly

the MGI) is not just about computational materials science.

In fact, it is mainly about experimental materials science,

from a practical perspective. It is often articulated by

computational simulation faculty who understand digital

workflows, perhaps fueling this misperception.

outside of the curriculum of university materials programs? What are elements of a curriculum to address twenty first century materials challenges? How should we balance coursework in conventional subjects with methods and tools that are responsive to ICME and the MGI?

Certainly, rigorous principles and foundations of MSE at the intersection of computation, experiment, and data science should be emphasized. The good news is that the modern curriculum can be updated rather than replaced. The more difficult news is that this update needs to be comprehensive in nature and reinforced throughout the curriculum and at various stages. Here are some simple suggestions relevant to educational curriculum for the future materials workforce:

- Materials characterization should be taught with modern data structures and formats in mind, e.g., hierarchical data format (HDF), ${ }^{27}$ including data registries, e.g., NIST ${ }^{11}$ and ecollaboration concepts. The value of metadata should be communicated.

- Increase faculty hires in computational thermodynamics, materials selection, and design to affect curriculum development to achieve greater impact across universities.

- Students should be exposed to systems engineering and design optimization concepts, including design of experiments. the bullet items listed above. Addressing UQ and its management in the context of systems engineering can dramatically accelerate materials innovation efforts. ${ }^{24}$ This was reinforced in the recent TMS study on Creating the Next-Generation Materials Genome Initiative Workforce. ${ }^{19}$ This study, funded by NSF DMREF and chaired by the author with 15 other contributing team members from academia, industry, and national laboratories, was undertaken against the backdrop of the significant strides made by NSF DMREF and other federal agency programs with input from the NSTC subcommittee of the MGI since the 2014 MGI strategic plan. ${ }^{3}$ Although a number of educational thrusts have emerged over the past decade, ${ }^{4,28}$ it was considered that future workforce issues have been given only cursory treatment in previous MGI-related efforts, necessitating an effort to broadly identify opportunities and provide guidance on next steps with respect to undergraduate and graduate education, postdoctoral training, and existing workforce professional opportunities. The final report of this effort is available for free download, ${ }^{19}$ and consists of an assessment of the current state of MGI education and training (60 of 114 undergrad/graduate materials programs in materials were assessed, including the alignment of their educational programs with MGI goals), results from a University Materials Council survey (academic unit 
heads) regarding the state of MGI education and perceived needs, assessment of knowledge and skills required for future MGI workforce, and barriers to adapting education and training for addressing MGI. A set of seven detailed action plans were presented.

\section{GEORGIA TECH'S INSTITUTE FOR MATERIALS: LESSONS LEARNED IN ADVANCING MATERIALS DATA SCIENCE}

Founded in 2012 as one of Georgia Tech's Interdisciplinary Research Institutes (IRIs), IMat $^{8}$ administers campus shared materials characterization facilities and serves the interests of well over 100 Georgia Tech faculty engaged in materialsrelated research and education. Having formed shortly after the launch of both ICME and the MGI, IMat's goals sought to add value to campus research capabilities and related educational initiatives, chiefly by focusing on new directions and methods to accelerate the pace of materials research and development. This has been pursued by organizing internal and external workshops, attracting cross-disciplinary research in materials data science across academic units and colleges, and exerting thought leadership on building a materials innovation ecosystem that fuses modern data science, modeling and simulation, manufacturing, entrepreneurship, and VVUQ methods to accelerate the pace of materials research, including both discovery and development phases/aspects.
Consonant with these goals, IMat has introduced the concept of a materials innovation ecosystem, which recognizes the pivotal role of contributions to the materials discovery and development enterprise by other engineering, science, computing, manufacturing, and business entities. As presented in Fig. 1,28 this is a key conceptual construct in pursuing materials data science within a university setting. The inner Venn diagram in Fig. 1 shows the overlap of experiments, computation, and digital data, as per the MGI strategic plan. ${ }^{3}$ It lies at the core of a multidisciplinary, distributed, collaborative network that couples materials development with manufacturing.

IMat has focused on seed fund support to foster research and development regarding key underdeveloped elements of the materials innovation ecosystem, ${ }^{4,28}$ including e-collaborative tools that focus on integration of materials data science and informatics with research efforts in various materials classes, decision support, and inverse methods for materials design exploration, high-throughput synthesis/processing-structure-property relations, materials information infrastructure, and education and training programs.

Foremost in addressing these gaps has been a two-pronged approach of educational materials: ecollaboration platforms and research support for graduate students to pursue work at the intersection of data science and materials. This has been spearheaded at Georgia Tech by the computational and data-driven body of work in both the Kali$\operatorname{dindi}^{29,30}$ and Ramprasad ${ }^{31}$ research groups. The corresponding Georgia Tech portfolio in Materials

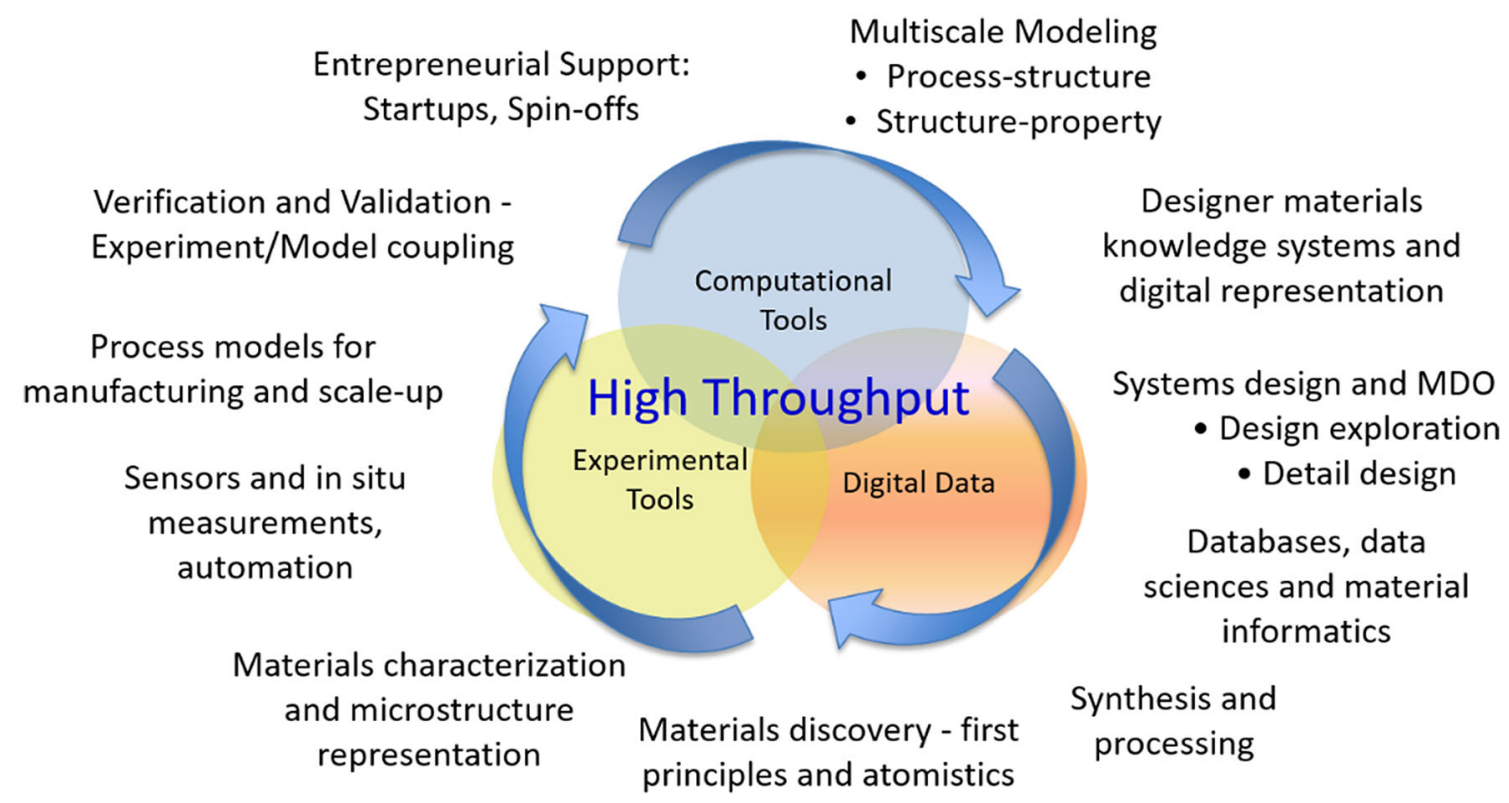

Distributed collaborative networks

Fig. 1. Cross-cutting cyber-physical materials innovation ecosystem that combines computation, experiment, and digital data via networking and engineering systems methods (reprinted from Ref. 28), built on the foundations of materials science and engineering. 
Data Science and Informatics is broad in scope and includes interactions in this domain with AFRL and NIST. From 2014 to 2019, the FLAMEL (From Learning, Analytics, and Materials to Entrepreneurship and Leadership) graduate student training program funded by the NSF supported over 24 thesis students paired from computing and materials research to integrate and explore various aspects of materials data science and informatics, with the goal of broadening participation and training in this regard. ${ }^{32}$ Students in this program were required to take a certain combination of core courses that cover fundamentals of materials science and engineering, mechanical and manufacturing sciences, and computer science (including data analytics). They were also required to take two courses aimed at synthesis and integration of these different disciplines. These integration courses are focused on materials informatics and are jointly taught by faculty from both materials science and computing and are offered through the College of Computing. Complementary programs at other universities include the Informatics Skunkworks at UW-Madison ${ }^{33}$ and DataEnabled Discovery and Design of Energy Materials (D3EM) at TAMU. ${ }^{34}$

Several other platforms that support future workforce development in materials data science were developed at Georgia Tech with support from IMat, including the Materials Knowledge Systems in Python (PyMKS) ${ }^{35}$ and the MATIN e-collaboration platform, ${ }^{36}$ both spearheaded by the Kalidindi group. Georgia Tech is a co-host institution for the NSF-funded South Big Data Innovation Hub funded by the NSF, ${ }^{37}$ with materials and manufacturing as an area of emphasis. The Georgia Tech NSF-supported HIVE computing cluster, ${ }^{38}$ attracted to address large-scale computing demands of astrophysics, computational biology, health sciences, computational chemistry, materials, and manufacturing, was completed in 2019 and is among the most powerful university-based supercomputers in the USA. The Ramprasad group at Georgia Tech introduced Khazana, ${ }^{39}$ a computational materials knowledgebase platform that includes polymer genomics. In addition to these supportive elements, textbooks and monographs are emerging that undergird foundations in some of the gap areas alluded to previously $\left(\mathrm{cf.}^{20,23,40}\right.$ ).

With support from IMat and the Provost, Georgia Tech developed and released two Massive Open Online Courses (MOOCs) on the Coursera platform: Materials Data Sciences and Informatics ${ }^{41}$ with over 9500 enrolled to date and Introduction to HighThroughput Materials Development, ${ }^{42}$ with over 7400 enrolled to date. We have found these MOOCs serve an important role in communicating the needs and value proposition for accelerated materials discovery and development, and as an enticement to draw interest from young prospective students as well as a point of reference for entry into industry skills retraining.

Of course, other universities have their own constraints and pathways to follow. Modern solutions consistent with the MGI and ICME for various application domains and materials classes are effectively pursued by fusing experiment and computation/theory with data science. ${ }^{43}$ There are a few key points we have learned from this experience that may offer utility for other universities and laboratories in tailoring their approaches to future workforce development and promulgation of materials data science:

- Don't start with faculty or senior staff in training and education, but rather with students or entry-level staff. Build interest and demonstrate value/capabilities, and this will serve to attract broader interest.

- Faculty will not effectively reinforce either computation or data science without incentives or coordination. Based on the 2019 UMC survey, ${ }^{19}$ $75 \%$ of respondent materials academic departments include experiments in capstone design, $54 \%$ have computation, but only $17 \%$ introduce data science. There is a long way to go.

- Identify, cultivate, and/or hire faculty leads, and then properly support and invest in their efforts. None of the significant progress achieved at Georgia Tech could have been realized without adding just a few key faculty members in materials informatics and machine learning. Sponsored research funding based on materials data science at Georgia Tech has grown by a factor of 20 or more since 2012.

- Rather than focusing on leadership, buy-in, and participation solely from materials-related academic departments (e.g., MSE, materials divisions in chemistry and/or physics), also work with various colleges (e.g., computer science, engineering, and sciences) and academic units (e.g., computational science and engineering, chemical and biomolecular engineering, mechanical engineering, aerospace engineering, industrial systems engineering, chemistry, physics, and biology).

- Support companion efforts in data science and informatics in computer science to co-develop courses focused on materials as well as hiring of faculty with interests and background in computational modeling of materials using various methods. Otherwise, computer science readily gravitates to big data attractors in finance, social systems, healthcare, etc.

\section{CONCLUSION}

Preparing the future materials workforce for revolutionary new trends in accelerated discovery, development, and deployment of new and improved 
materials demands emphasis on high-throughput computational and experimental methods, coupled with modern data science. Challenges in closing gaps within our established approaches and infrastructure are substantial; it is logical to address them within the vision of a cross-cutting materials innovation ecosystem and supportive infrastructure. We have focused on the intersection of materials and manufacturing, and have identified substantial gaps in our curriculum related to instruction and reinforcement of basic concepts underlying uncertainty quantification and data science. Systems engineering is too often shortchanged in the educational experience in materials education. Additive manufacturing and the concept of a digital twin are highlighted as two examples of emergent application domains that capture that imagination; for both, foundational principles relevant to a wide range of materials classes can be communicated in a vertically integrated manner through the curriculum, including capstone design. This also reinforces systems engineering thinking. It is recommended to build on digital, web-based instructional portals, including modules developed by leaders in the MGI and ICME that can be flexibly integrated and broadly employed, to provide a more robust infrastructure with reinforcement throughout the curriculum. This lends itself naturally to acculturation of digital workflows and e-collaboration. The article closes with a brief discussion and recommendations based on our experience at Georgia Tech over the past decade in supporting, advancing, and disseminating basic concepts and principles of materials data science in research and education.

\section{ACKNOWLEDGEMENTS}

The author is grateful to the office of the Executive Vice President of Research at Georgia Tech for standing up the Institute for Materials at Georgia Tech as an outcome of a campus-wide materials strategic planning exercise in 2011. IMat support has been instrumental in framing and pursuing the vision of a materials innovation ecosystem that resonates with aspirations of the MGI and ICME. Special thanks go to Eric Vogel and W. Jud Ready, Associate Directors since IMat's inception, longtime center manager Cecelia Jones, and MGI strategists Surya Kalidindi and Rampi Ramprasad, already mentioned in the text. The author also wishes to acknowledge various external colleagues and thought leaders in this regard, including members of the TMS Materials Innovation Committee, Jim Warren at NIST, Chuck Ward at AFRL, and the wise counsel and longtime support of former and current IMat External Advisory Panel members not already mentioned (Ian Robertson, University of Wisconsin; Terrence Aselage, Sandia; Jerry Young, Boeing; Rusty Gray, Los Alamos; Javier Llorca, IMDEA Materials Institute in Madrid; Harald Roeckel, BASF; Todd Summe, Novelis; L. Cate
Brinson, Duke University; Edgar Lara Curzio, ORNL; Mike Valley, Sandia; Raymundo Arroyave, Texas A\&M; Mike Pcolinski, BASF; Matt Miller, Cornell University; Teresa Kotanchek, Evolved Analytics, Miquel Salmeron, LBNL). We also acknowledge important collaborations with likeminded colleagues involved with university materials research and education transformation at the University of Wisconsin (Dane Morgan and Tom Kuech), the University of Michigan (John Allison and Katsuyo Thornton), and Texas A\&M (Raymundo Arroyave). Members of the TMS study on the future MGI workforce are gratefully acknowledged. ${ }^{19}$ Finally, the author acknowledges the support of the Carter N. Paden, Jr. Distinguished Chair in Metals Processing.

\section{REFERENCES}

1. T.M. Pollock (Chair) and J.E. Allison (Vice Chair), Integrated Computational Materials Engineering: A Transformational Discipline for Improved Competitiveness and National Security, Committee on Integrated Computational Materials Engineering, National Materials Advisory Board, Division of Engineering and Physical Sciences, National Research Council of the National Academies, (Washington, D.C.: National Academies Press, 2008).

2. National Science and Technology Council, Executive Office of the President of the United States, Materials Genome Initiative for Global Competitiveness (National Science and Technology, Council, 2011). https://mgi.gov/sites/default/file s/documents/materials_genome_initiative-final.pdf. Accessed 29 June 2020.

3. National Science and Technology Council, Executive Office of the President of the United States, Materials Genome Initiative Strategic Plan (Committee on Technology, Subcommittee on the Materials Genome Initiative, December 2014). https://mgi.gov/sites/default/files/documents/mgi_stra tegic_plan_-_dec_2014.pdf. Accessed 29 June 2020.

4. D.L. McDowell and S.R. Kalidindi, MRS Bull. 41, 326 (2016).

5. X. Liu, D. Furrer, J. Kosters, and J. Holmes, Vision 2040: A Roadmap for integrated, multiscale modeling and simulation of materials and systems, NASA/CR-2018-219771, 2018. https://ntrs.nasa.gov/archive/nasa/casi.ntrs.nasa.gov/20180 002010.pdf. Accessed 28 June 2020.

6. D.L. McDowell, W.J. Ready, D.D. Morgan, T.F. Kuech and J.E. Allison, Workshop Report: Building an Integrated Materials Genome Initiative Accelerator Network, (Workshop on Building an Integrated Materials Genome Initiative Accelerator Network, Atlanta, GA, June 2014). http://mate rials.gatech.edu/sites/default/files/documents/accelerator_n etwork_report.final_.pdf. Accessed 29 June 2020.

7. U.S. News and World Report, Best Materials Engineering Programs, (2020). https://www.usnews.com/best-graduate-s chools/top-engineering-schools/material-engineering-rankin gs. Accessed 28 June 2020

8. Georgia Tech Institute for Materials (2020). http://www.ma terials.gatech.edu/. Accessed 28 June 2020.

9. Wisconsin Materials Institute (2020). https://materials.wis c.edu/. Accessed 28 June 2020.

10. The Materials Project (2020). https://www.materialsproject. org. Accessed 29 June 2020.

11. National Institute of Standards and Technology, Materials Genome Initiative (NIST, 2020). https://www.nist.gov/mgi. Accessed 28 June 2020.

12. AMFG Autonomous Manufacturing, Industry 4.0: RealWorld Examples of Digital Manufacturing in Action, (Autonomous Manufacturing Ltd, London, UK, 2020). https://a mfg.ai/2019/03/28/industry-4-0-7-real-world-examples-of-di gital-manufacturing-in-action/. Accessed 2 July 2020. 
13. J.H. Panchal, S.R. Kalidindi, and D.L. McDowell, Comput. Aided Des. 45, 4 (2013).

14. S.A.H. Motaman, F. Kies, P. Köhnen, M. Letang, M. Lin, A. Molotnikov, and C. Haase, JOM 72, 1092 (2020).

15. C.B. Williams, T.W. Simpson and M. Hripko, Advancing the Additive Manufacturing Workforce: Summary and Recommendations from a NSF Workshop. in Proceedings of the ASME 2015 International Design Engineering Technical Conferences and Computers and Information in Engineering Conference. Volume 3: 17th International Conference on Advanced Vehicle Technologies; 12th International Conference on Design Education; 8th Frontiers in Biomedical Devices. Boston, MA, USA. August 2-5, 2015. V003T04A003. ASME. https://doi.org/10.1115/DETC2015-47274.

16. E. Glaessgen and D. Stargel, D., The Digital Twin Paradigm for Future NASA and U.S. Air Force Vehicles, 53rd AIAA/ ASME/ASCE/AHS/ASC Structures, Structural Dynamics and Materials Conference, Honolulu, HI, 2012, AIAA 20121818. http://dx.doi.org/10.2514/6.2012-1818, https://ntrs.nas a.gov/archive/nasa/casi.ntrs.nasa.gov/20120008178.pdf.

17. E.J. Tuegel, A.R. Ingraffea, T.G. Eason, and S.M. Spottswood, Int. J. Aerospace Eng. 2011, 154798 (2011).

18. D.L. McDowell, Revolutionising Product Design and Performance with Materials Innovation, The Next Production Revolution, Implications for Government and Business, Ch. 6, 215 (Paris: OECD Publishing, 2017). http://www.oecd.org/ sti/ind/next-production-revolution.htm. Accessed 28 June 2020 .

19. D.L. McDowell, R. Arroyave, A. Beese, M. Earnest, A.G. Kusne, R. Ramprisad, X. Sun, C. Tway, K. Anderson, M. Asta, S. Curtarolo, W. Joost, M. Manuel, G. Rodriguez, K. Thornton, and P. Voorhees, Creating the Next-Generation Materials Genome Initiative Workforce (The Minerals, Metals \& Materials Society (TMS), Pittsburgh, PA 2019). Electronic copies available at www.tms.org/MGIworkforce.

20. D.L. McDowell, J.H. Panchal, H.-J. Choi, C.C. Seepersad, J.K. Allen, and F. Mistree, Integrated Design of Multiscale, Multifunctional Materials and Products, 1st ed. (Elsevier: Butterworth-Heinemann, 2010).

21. T. Lookman, P.V. Balachandran, D. Xue, and R. Yuan, NPJ Comput Mater 5, 21 (2019).

22. Y. Liu, T. Zhao, W. Ju, and S. Shi, J. Materiomics 3, 159 (2017).

23. Y. Wang and D.L. McDowell, Uncertainty quantification in materials modeling. in Uncertainty in Multiscale Materials Modeling, ed. by Y. Wang and D.L. McDowell (Cambridge, MA: Elsevier Series in Mechanics of Advanced Materials, Woodhead, 2020), p. 1.

24. W. Oberkampf, D. Fullwood, K. Garikipati, M. Koslowski, S. Mahadevan, A. Masud, D.L. McDowell, P. Perdikaris, C. Reina, W. Rider, K. Solanki, and M. Tonks, Accelerating the Broad Implementation of Verification \& Validation in Computational Models of the Mechanics of Materials and Structures, (The Minerals, Metals \& Materials Society (TMS), Pittsburgh, PA 2020). https://www.tms.org/portal/P UBLICATIONS/Studies/VV2/portal/Publications/Studies/V V2/vv2.aspx?hkey=a522173d-cbe6-425c-b1fd-5db9c111d9d6. Accessed 29 June 2020.

25. Project Jupyter (2020). https://jupyter.org/. Accessed 29 June 2020.
26. Python (2020). https://www.python.org/. Accessed 20 Sept 2020.

27. The HDF Group, The HDF5 Library \& File Format (2020). h ttps://www.hdfgroup.org/solutions/hdf5/. Accessed 20 Sept 2020.

28. S.R. Kalidindi, A.J. Medford, and D.L. McDowell, JOM 68, 2126 (2016).

29. S.R. Kalidindi and M. De Graef, Annu. Rev. Mater. Res. 45, 171 (2015)

30. Materials Informatics for Engineering Design, Surya Kalidindi Research Group, Georgia Tech (2020). https://matin.ga tech.edu/groups/mined_super/research. Accessed 19 Sept 2020.

31. Ramprasad Group, Georgia Tech (2020). http://ramprasad. mse.gatech.edu/. Accessed 29 June 2020.

32. From Learning, Analytics, and Materials to Entrepreneurship and Leadership Doctoral Traineeship Program, Georgia Tech (2020). http://flamel.gatech.edu. Accessed 28 June 2020.

33. Informatics Skunkworks, University of Wisconsin-Madison (2020). https://skunkworks.engr.wisc.edu/. Accessed 19 Sept 2020.

34. Data-Enabled Discovery and Design of Energy Materials, Texas A\&M (2020). https://d3em.tamu.edu/. Accessed 19 Sept 2020.

35. Materials Knowledge Systems in Python (2020). http://mate rialsinnovation.github.io/pymks/. Accessed 29 June 2020.

36. Materials Innovation Network, Georgia Tech (2020). https:// matin.gatech.edu/. Accessed 19 Sept 2020.

37. South Big Data Innovation Hub (2020). https://southbigda tahub.org/about-this-blog/. Accessed 29 June 2020.

38. Hive Supercomputer Makes its Debut, Georgia Tech College of Computing News (November 19, 2019). https://www.cc.ga tech.edu/news/629130/hive-supercomputer-makes-its-debu t. Accessed 19 Sept 2020.

39. Khazana, Materials Data and Tools from the Ramprasad Group (2020). https://khazana.gatech.edu/. Accessed 19 Sept 2020.

40. S.R. Kalidindi, Hierarchical Materials Informatics, 1st ed. (Oxford: Butterworth-Heinemann, 2015).

41. Materials Data Sciences and Informatics, Coursera (Massive Open Online Course offered by Georgia Tech, 2020). h ttps://www.coursera.org/learn/material-informatics. Accessed 20 Sept 2020

42. Introduction to High-Throughput Materials Development, Coursera (Massive Open Online Course offered by Georgia Tech, 2020). https://www.coursera.org/learn/high-throughp ut. Accessed 20 Sept 2020.

43. J.J. de Pablo, N.E. Jackson, M.A. Webb, L.-Q. Chen, J.E. Moore, D. Morgan, R. Jacobs, T. Pollock, D.G. Schlom, E.S. Toberer, J. Analytis, I. Dabo, D.M. DeLongchamp, G.A. Fiete, G.M. Grason, G. Hautier, Y. Mo, K. Rajan, E.J. Reed, E. Rodriguez, V. Stevanovic, J. Suntivich, K. Thornton, and J.-C. Zhao, NPJ Comput. Mater. 5, 41 (2019).

Publisher's Note Springer Nature remains neutral with regard to jurisdictional claims in published maps and institutional affiliations. 